Vol. 4, No. 2 | Jul - Dec 2020

\title{
On Study Of Generalized Nonlinear Black Scholes Equation By Reduced Differential Transform Algorithm
}

\author{
Naresh Kumar Solanki ${ }^{1}$, Syed Feroz Shah ${ }^{1}$
}

\begin{abstract}
:
The objective of the work is essential to construct an approximate solution of the generalization of nonlinear Black-Scholes partial differential equation, modeling price slippage impact of transaction coast option, through promising computational algorithm called Reduced Differential Transform Algorithm. This work also shows that the algorithm can be efficiently employed to construct explicit solutions highly nonlinear equations arising in the financial market. We have also shown a graphical behavior of the constructed solutions.
\end{abstract}

Keywords: Nonlinear Black-Scholes model, PDE, differential transform algorithm

\section{Introduction}

This paper is intended to construct an approximate and closed-form solution to the following initial value problem,

$$
\begin{gathered}
\frac{\partial u}{\partial t}+\frac{1}{2} \sigma^{2} s^{2} \frac{\partial^{2} u}{\partial s^{2}}\left(1+2 \rho s \frac{\partial^{2} u}{\partial s^{2}}\right)+r s \frac{\partial u}{\partial s} \\
-r u=0
\end{gathered}
$$

subject to the initial condition,

$$
\begin{array}{r}
u(s, 0)=\frac{1}{\rho}\left[\frac{-4}{\sigma^{2}}(r-c) \sqrt{K s} e^{\frac{-c}{2} T}+\left(\frac{r-c}{\sigma^{2}}+\frac{1}{4}\right)(1-\ln s) s\right. \\
\left.+\frac{(r-c)}{\sigma^{2}} K\left(e^{-c T}-(r-c) e^{-r t}\right)\right]
\end{array}
$$

Where $S$ represents the stock price, $\rho \geq 0$ it shows the measure of liquidity of the market, and also represents volatility as well. $u(s, t)$ represents the option price and it's a measure of the price of slippage impact of a trade felt by all participants of a market.

\section{Description of Differential Transform Algorithm}

This section has been dedicated to give a precise description of the Reduced Differential Transform algorithm and how it works. Assume that we have a function $u(x, t)$ with arguments $\mathrm{x}$ and $\mathrm{t}$, that can express as the product two functions of $x$ and $t$ i.e. i.e., $u(x, t)=f(x) g(t)$. Then differential transform of the function $u(x, t)$ can be explicitly written as,

$$
u(x, t)=\left(\sum_{i=0}^{\omega} F(i) x^{i}\right)\left(\sum_{i=0}^{\infty} G(i) t^{i}\right)=\sum_{k=0}^{\infty} U_{k}(x) t^{k},
$$

where $U_{k}(x)$ is transformed function in $\mathrm{x}$.

The more careful and precise definitions of transform of function $u(x, t)$ is following, (cf. ${ }^{14-16}$ ).

Definition: Consider a function $u(x, t)$ is call option where $\mathrm{u}$ represents the price of option and $\mathrm{t}$ represents the time $t \geq 0$ and

${ }^{1}$ Basic Science and related science MUET, Jamshoro, Pakistan

Corresponding Author: feroz.shah@ faculty.muet.edu.pk 
Naresh Kumar (et al.), On Study Of Generalized Nonlinear Black Scholes Equation By Reduced Differential Transform Algorithm

space $x \in \mathbb{R}$. Then define the transform of $u(x, t)$ as,

$$
U_{k}(x)=\frac{1}{k !}\left[\frac{\partial^{k}}{\partial t^{k}} u(x, t)\right]_{t=0} .
$$

$$
\begin{aligned}
(k+1) U_{k+1}(x) & \\
& =G_{k}(x)-R U_{k}(x) \\
& -N U_{k}(x)
\end{aligned}
$$

where the $U_{k}(x)$ can be treated as transformed $u(x, t)$, and is essentially analogous to Taylor's coefficient in the 2D Taylor expansion.

To recover the function $u(x, t)$ from transformed functions $U_{k}(x)$, we define the following inverse of differential transform in the following manner.

Definition: Consider a function $u(x, t)$ is call option where $u$ represents the price of option and $t$ represents the time $t \geq 0$, Then we can define the transform of $u(x, t)$ as, Then define the transform of $U_{k}(x)$, as,

$u(x, t)=\sum_{k=0}^{\infty} U_{k}(x) t^{k}$

Or more explicitly,

$$
u(x, t)=\sum_{k=0}^{\infty} \frac{1}{k !}\left[\frac{\partial^{k}}{\partial t^{k}} u(x, t)\right]_{t=0} t^{k}
$$

where $U_{k}(x), R U_{k}(x), N U_{k}(x)$ and $G_{k}(x)$ denotes the differential transformation of $\operatorname{Lu}(x, t), \operatorname{Ru}(x, t), N u(x, t) \quad$ and $\quad g(x, t)$ respectively. Hence the key computation that one need to is the computation of functions $U_{1}, U_{2}, U_{3} \ldots$ through recursive relation (4), by choosing $U_{0}(x)=f(x)$.

Once $U_{1}, U_{2}, U_{3} \ldots U_{n}$ are found then we can write n-term approximate solution of PDE as follows:

$$
\tilde{u}_{n}(x, t)=\sum_{k=0}^{n} U_{k}(x) t^{k} .
$$

Thus, by increasing $\mathrm{n}$ more and more we get an exact solution of nonlinear PDE (4),

$$
\tilde{u}(x, t)=\lim _{n \rightarrow \infty} \sum_{k=0}^{n} U_{k}(x) t^{k}
$$

Next, we discuss how the above-described transformations can be implemented to solve the concrete nonlinear partial differential equations. Consider a nonlinear PDE in its generalized form,

$$
\begin{gathered}
L u(x, t)+R u(x, t)+N u(x, t) \\
=g(x, t)
\end{gathered}
$$

Subject to the initial condition $u(x, 0)=$ $f(x)$. Here $L$ denotes an operator $\frac{\partial}{\partial t}, R u(x, t)$ denotes the linear part of PDE that contains the linear expressions of $u$ and its derivatives, $N u(x, t)$ denotes the operator/expression containing the nonlinear terms involving $u$, and its derivatives operator, $g(x, t)$ stands for an in-homogeneous term that can be treated a forcing factor in the model. Taking the differential transform of the equation (3) leads to the following recursive relation,

Based on the definition of the reduced differential transform algorithm following table of transformations (see next page) can be proved. For the readers interested in the proofs we refer to [14], [15], and [16].

\footnotetext{
Sukkur IBA Journal of Computing and Mathematical Science - SJCMS | Vol. 4 No. 2 July - December 2020 ๑ Sukkur IBA University
} 
Table 1 Reduced differential transformation

\begin{tabular}{ll}
\hline Functional form & Transformed form \\
\hline$u(x, t)$ & $U_{k}(x)=\frac{1}{k !}\left[\frac{\partial^{k}}{\partial t^{k}} u(x, t)\right]_{t=0}$ \\
$w(x, t)=u(x, t) \pm v(x, t)$ & $W_{k}(x)=U_{k}(x) \pm V_{k}(x)$ \\
$w(x, t)=\alpha u(x, t)$ & $W_{k}(x)=\alpha U_{k}(x)(\alpha$ is a constant) \\
$w(x, t)=x^{m} t^{n}$ & $W_{k}(x)=x^{m} \delta(k-n), \quad \delta(k)= \begin{cases}1, & k=0 \\
0, & k \neq 0\end{cases}$ \\
$w(x, t)=x^{m} t^{n} u(x, t)$ & $W_{k}(x)=x^{m} U_{k-n}(x)$ \\
$w(x, t)=u(x, t) v(x, t)$ & $W_{k}(x)=\sum_{r=0}^{k} V_{r}(x) U_{k-r}(x)=\sum_{r=0}^{k} U_{r}(x) V_{k-r}(x)$ \\
$w(x, t)=\frac{\partial^{r}}{\partial t^{r}} u(x, t)$ & $W_{k}(x)=(k+1) \cdots(k+r) U_{k+r}(x)=\frac{(k+r) !}{k !} U_{k+r}(x)$ \\
$w(x, t)=\frac{\partial}{\partial x} u(x, t)$ & $W_{k}(x)=\frac{\partial}{\partial x} U_{k}(x)$ \\
\hline
\end{tabular}

\section{The solution of Generalized form of nonlinear Black-Scholes equation by Reduced Differential Transform Algorithm}

For following the Nonlinear Black-Scholes equation, we are applying the RDTM method to get an approximate solution.

Let us restart by rewriting the equation (1) in the following form,

$$
\begin{gathered}
\frac{\partial u}{\partial t}=-\left[\frac{\sigma^{2} s^{2}}{2} \frac{\partial^{2} u}{\partial s^{2}}\left(1+2 \rho s \frac{\partial^{2} u}{\partial s^{2}}\right)+r s \frac{\partial u}{\partial s}\right. \\
-r u]
\end{gathered}
$$

On the application of RDTM on the above last equation, using Table 1of transforms, we get

$$
\begin{aligned}
(k+1) u_{k+1}=- & {\left[\frac{1}{2} \sigma^{2} s^{2} \frac{\partial^{2} u_{k}}{\partial s^{2}}\right.} \\
& +\rho \sigma^{2} s^{3}\left(\frac{\partial^{2} u_{k}}{\partial s^{2}}\right)^{2} \\
& \left.+r s \frac{\partial u_{k}}{\partial s}-r u_{k}\right]
\end{aligned}
$$

where $u_{k}(x)$ is transformed function and dimensional spectrum function is $t$,

$$
A_{k}=\sum_{k=0}^{n} \frac{\partial^{2} u(s, k)}{\partial s^{2}} \frac{\partial^{2} u(s, h-k)}{\partial s^{2}}
$$

Let us start by computing the $u_{1}$. The explicit expression for it can be obtained by setting $k=0$ in (9), as follows,

$$
\left.\begin{array}{r}
u_{1}=-\left[\frac{1}{2} \sigma^{2} s^{2} \frac{\partial^{2} u_{0}}{\partial s^{2}}+\rho \sigma^{2} s^{3} A_{0}\right. \\
+r s \frac{\partial u_{0}}{\partial s}-r u_{0}
\end{array}\right]
$$

It is clear that to compute the above expression we need values of $A_{0}, \frac{\partial u_{0}}{\partial s}$ and $\frac{\partial^{2} u_{0}}{\partial s^{2}}$. From the initial condition equation (9), we solve the first and second partial derivatives of equation (9), we have

$$
\begin{aligned}
\frac{\partial u_{0}}{\partial s}=\frac{1}{\rho}\left[\frac{-2}{\sigma^{2}}(r\right. & -c) \frac{\sqrt{K}}{\sqrt{s}} e^{\frac{-c}{2} T} \\
& -\operatorname{lns}\left(\frac{(r-c)}{\sigma^{2}}\right. \\
& \left.\left.+\frac{1}{4}\right)\right]
\end{aligned}
$$


Naresh Kumar (et al.), On Study Of Generalized Nonlinear Black Scholes Equation By Reduced Differential Transform Algorithm

$$
\begin{aligned}
\frac{\partial^{2} u_{0}}{\partial s^{2}}=\frac{1}{\rho}\left[\frac{(r-c)}{\sigma^{2}}\right. & \frac{\sqrt{K}}{s^{\frac{3}{2}}} e^{\frac{-c}{2} T} \\
& -\frac{1}{s}\left(\frac{(r-c)}{\sigma^{2}}\right. \\
& \left.\left.+\frac{1}{4}\right)\right]
\end{aligned}
$$

Now taking $k=0$ in Equation (9) and plugging partial derivatives from (10) and (11), we get the following value of $A_{0}$

$A_{0}=\left(\frac{\partial^{2} u_{0}}{\partial s^{2}}\right)^{2}$

$=\frac{1}{\rho^{2}}\left[\frac{(r-c)^{2}}{\sigma^{4}} \frac{\mathrm{K}}{s^{3}} e^{-\mathrm{c} T}\right.$

$-2\left(\frac{(r-c)}{\sigma^{2}} \frac{\sqrt{K}}{s^{\frac{3}{2}}} e^{\frac{-c T}{2}}\right)\left(\frac{1}{s}\left(\frac{(r-c)}{\sigma^{2}}+\frac{1}{4}\right)\right)$

$\left.+\frac{1}{\mathrm{~s}^{2}}\left(\frac{(r-c)}{\sigma^{2}}+\frac{1}{4}\right)^{2}\right]$

On substituting values $A_{0}, \frac{\partial u_{0}}{\partial s}$ and $\frac{\partial^{2} u_{0}}{\partial s^{2}}$ in expression for $u_{1}$ and simplifying we obtain

$$
\begin{array}{r}
u_{1}=\frac{1}{\rho}\left[-\frac{2 c(r-c)}{\sigma^{2}} \sqrt{K s} e^{\frac{-c}{2}}-\frac{(r-c)^{2}}{\sigma^{2}} K e^{-c T^{T}}-\frac{\sigma^{2} s}{2}\left(\frac{(r-c)^{2}}{\sigma^{4}}-\frac{1}{16}\right)\right. \\
\left.-r s\left(\frac{r-c}{\sigma^{2}}+\frac{1}{4}\right)+\frac{r(r-c)}{\sigma^{2}} K e^{-c r^{T}}-r(r-c) e^{-r t}\right]
\end{array}
$$

Next, let us move towards computing the valued $u_{2}$. To do so let us substitute $k=1$ in recursive relation $(8)$,

$u_{2}=-\left[\frac{1}{2} \sigma^{2} s^{2} \frac{\partial^{2} u_{1}}{\partial s^{2}}+\rho \sigma^{2} s^{3} A_{1}+r s \frac{\partial u_{1}}{\partial s}-r u_{1}\right]$ Therefore, to compute $u_{2}$, we need to explicitly find $A_{1}, \frac{\partial u_{1}}{\partial s}$ and $\frac{\partial^{2} u_{1}}{\partial s^{2}}$. Using equation (13), we may find the partial derivatives as following,

$$
\begin{gathered}
\frac{\partial u_{1}}{\partial s}=\frac{1}{\rho}\left[\frac{-\mathrm{c}(r-c)}{\sigma^{2}} \frac{\sqrt{K}}{s^{\frac{1}{2}}} e^{\frac{-c}{2} T}\right. \\
-\sigma^{2}\left(\frac{(r-c)^{2}}{\sigma^{4}}-\frac{1}{16}\right) \\
\left.+r\left(\frac{r-c}{\sigma^{2}}+\frac{1}{4}\right)\right]
\end{gathered}
$$

$\frac{\partial^{2} u_{1}}{\partial s^{2}}=\frac{1}{\rho}\left[\frac{\mathrm{c}(r-c)}{2 \sigma^{2}} \frac{\sqrt{K}}{s^{\frac{3}{2}}} e^{\frac{-c}{2} T}\right]$

On employing (9), (14), and (15), we can explicitly compute $A_{1}$ as,

$$
\begin{aligned}
& A_{1}=\frac{1}{\rho^{2}}\left[\frac{c(r-c)^{2}}{\sigma^{4}} \frac{\mathrm{K}}{s^{3}} e^{-c T}\right. \\
&- \frac{\mathrm{c}(r-c)}{\sigma^{2}}\left(\frac{(r-c)}{\sigma^{2}}\right. \\
&\left.\left.+\frac{1}{4}\right) \frac{\sqrt{K}}{s^{\frac{5}{2}}} e^{\frac{-c}{2} T}\right] \\
& A_{1}=2 \frac{\partial^{2} u_{0}}{\partial s^{2}} \frac{\partial^{2} u_{1}}{\partial s^{2}}
\end{aligned}
$$

Now we are in a position to compute $u_{2}$ using the values of $A_{1}, \frac{\partial u_{1}}{\partial s}$ and $\frac{\partial^{2} u_{1}}{\partial s^{2}}$ from (16), (14), (15) respectively,

$$
\begin{gathered}
u_{2}=\frac{1}{\rho}\left[-\frac{c^{2}(r-c)}{2 \sigma^{2}} \sqrt{K s} e^{\frac{-c}{2} T}-\frac{c(r-c)^{2}}{2 \sigma^{2}} K e^{-c T}-\frac{r(r-c)^{2}}{2 \sigma^{2}} K e^{-c T}\right. \\
\left.+\frac{r^{2}(r-c)}{2 \sigma^{2}} K e^{-c T}-\frac{r^{2}(r-c)^{2}}{2 \sigma^{2}} K e^{-r t}\right]
\end{gathered}
$$

Finally, we aim to compute $u_{3}$. To do, by substituting $k=2$ in recursive relation (8) we get,

$3 u_{3}=-\left[\frac{1}{2} \sigma^{2} s^{2} \frac{\partial^{2} u_{2}}{\partial s^{2}}+\rho \sigma^{2} s^{3} A_{2}+r s \frac{\partial u_{2}}{\partial s}-r u_{2}\right]$

Hence to compute $u_{3}$, we need to compute $A_{2}, \frac{\partial u_{2}}{\partial s}$ and $\frac{\partial^{2} u_{2}}{\partial s^{2}}$. The partial derivatives of $u_{2}$ can be computed using equation (17),

$$
\frac{\partial u_{2}}{\partial s}=\frac{1}{\rho}\left[-\frac{c^{2}(r-c)}{4 \sigma^{2}} \frac{\sqrt{K}}{\sqrt{s}} e^{\frac{-c}{2} T}\right]
$$




$$
\frac{\partial^{2} u_{2}}{\partial s^{2}}=\frac{1}{\rho}\left[\frac{\mathrm{c}^{2}(r-c)}{8 \sigma^{2}} \frac{\sqrt{K}}{s^{\frac{3}{2}}} e^{\frac{-c}{2} T}\right]
$$

Next, an explicit expression for $A_{2}$ can be obtained by putting $k=2$ in (9),

$$
A_{2}=\frac{\partial^{2} u_{0}}{\partial s^{2}} \frac{\partial^{2} u_{2}}{\partial s^{2}}+\frac{\partial^{2} u_{1}}{\partial s^{2}} \frac{\partial^{2} u_{1}}{\partial s^{2}}+\frac{\partial^{2} u_{2}}{\partial s^{2}} \frac{\partial^{2} u_{0}}{\partial s^{2}}
$$

Using (11), (15), and (17) in the above, and we get,

$$
A_{2}=\frac{1}{\rho^{2}}\left[\left(\frac{c^{2}(r-c)^{2}}{2 \sigma^{4}} \frac{K}{s^{3}} e^{-c T}-\left(\frac{c^{2}(r-c)}{4 \sigma^{2}} \frac{\sqrt{K}}{s^{\frac{5}{2}}} e^{\frac{-c}{2}} T\right)\left(\frac{(r-c)}{\sigma^{2}}+\frac{1}{4}\right)\right)\right]
$$

Now employing the $A_{2}, \frac{\partial u_{2}}{\partial s}$ and $\frac{\partial^{2} u_{2}}{\partial s^{2}}$ from (20), (18), and (19), in $u_{3}$, we get,

$$
\begin{aligned}
& u_{3}=\frac{1}{\rho}\left[-\frac{c^{3}(r-c)}{4.3 \sigma^{2}} \sqrt{K s} e^{\frac{-c}{2} T}-\frac{c^{2}(r-c)^{2}}{3.2 \sigma^{2}} K e^{-c T}-\frac{c r(r-c)^{2}}{3.2 \sigma^{2}} K e^{-c T}-\right. \\
& \left.\frac{r^{2}(r-c)^{2}}{3.2 \sigma^{2}} K e^{-c T}+\frac{r^{3}(r-c)}{3.2 \sigma^{2}} K e^{-c T}-\frac{r^{3}(r-c)^{2}}{3.2 \sigma^{2}} K e^{-r t}\right]
\end{aligned}
$$

Finally, the approximate solution to our main problem can be given as,

$$
u(x, t)=\sum_{k=0}^{\infty} U_{k}(x) t^{k} \approx u_{0}+u_{1} t+u_{2} t^{2}+u_{3} t^{3}
$$

On substituting, values of

$u_{0}, u_{1}, u_{2}$ and $u_{3}$ from (13), (17), and (20) in the last equation, we get the following closed-form approximate solution of problem (1),

The 3D graph of the solution has been computed using Mathematica,

$$
\begin{aligned}
& u(s, t) \approx \frac{1}{\rho}\left[\frac{-4}{\sigma^{2}}(r-c) \sqrt{K s} e^{\frac{-c}{2}(T-t)}+\left(\frac{r-c}{\sigma^{2}}+\frac{1}{4}\right)(1-\ln s) s\right. \\
& \left.+\left(\frac{\sigma^{2}+4 r}{16}+\frac{c(r-c)}{\sigma^{2}}\right) s t+\frac{(r-c)}{\sigma^{2}} K\left(e^{-c(T-t)}-(r-c) e^{-r(T-t)}\right)\right]
\end{aligned}
$$

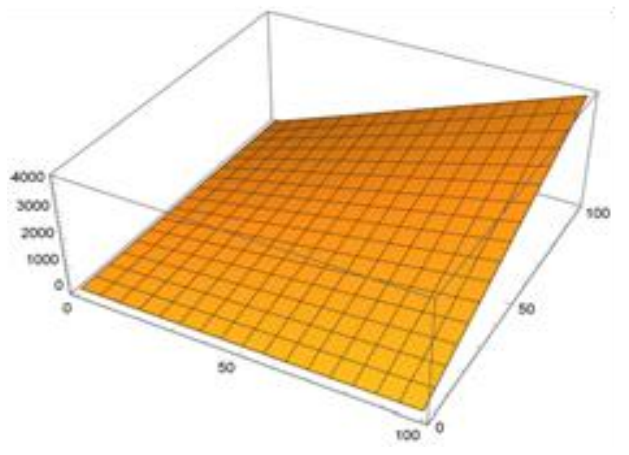

\section{Conclusion}

Transaction cost Black-Scholes model is highly nonlinear in its form. In this article, we have computed an explicit approximation solution to a highly nonlinear version of the generalized nonlinear Black-Scholes equation through a highly efficient algorithm known as the reduced differential transform method. This solution obtained can be used for pricing European call and put option at time $t \geq 0$, and when $\mathrm{c} \neq \mathrm{r}$. Our work shows that Reduced differential transform methods are very convenient for constructing the explicit scheme of higher nonlinear problems arising in the financial world.

\section{References}

[1] B. Lin and Z. Yin, "The Cauchy problem for a generalized Camassa-Holm equation with velocity potential.," Applicable Analysis, vol. 97, no. 3, pp. 354-367, 2018.

[2] R. Camassa and a. D. Holm, "An integrable shallow water equation with peaked solitons," Physical review letters, vol. 71, pp. 1661-1664, 1993.

[3] C. A, "On the scattering problem for the Camassa--Holm equation," Proceedings of the Royal Society of London. Series A: Mathematical, Physical and Engineering Sciences, vol. 457, pp. 953-970, 2001.

[4] Y. Keskin and G. Oturanc, "Reduced differential transform method for

\footnotetext{
Sukkur IBA Journal of Computing and Mathematical Science - SJCMS | Vol. 4 No. 2 July - December 2020 @ Sukkur IBA University
} 
Naresh Kumar (et al.), On Study Of Generalized Nonlinear Black Scholes Equation By Reduced Differential Transform Algorithm (рp. 22 - 27)

generalized KDV equations,"

Mathematical and Computational applications, vol. 15, pp. 382-393, 2010.

[5] Y. Keskin and G. Oturan, "Application of reduced differential transformation method for solving gas dynamics equation," International Journal of Contemporary Mathematical Sciences, vol. 22, pp. 1091-1096, 2010.

[6] Y. Keskin and G. Oturanc, "Reduced differential transform method for solving linear and nonlinear wave equations," 2010.

[7] Zheng, R. a. Yin and Zhaoyang, "Global weak solutions for a generalized Novikov equation," Monatshefte fur Mathematik, vol. 188, pp. 387-400, 2019.

[8] Novikov and Vladimir, "Generalizations of the Camassa-Holm equation.," Journal of Physics A: Mathematical and Theoretical, vol. 42, p. 342002, 2009.

[9] Henry and D., "Infinite propagation speed for the Degasperis-Procesi equation," Journal of mathematical analysis and applications, vol. 311, pp. 755-759, 2005.

[10] Lenells and Jonatan, "Travelling wave solutions of the Degas," Journal of mathematical analysis and applications, vol. 306, pp. 72-82, 2005.

[11] Oclite, G. M. a. Karlsen and K. H, "On the well posedness of the DegasperisProcesi equation," Journal of Functional Analysis, vol. 233, pp. 6091, 2006.

[12] Degasperis, A. a. Procesi and Michela, "Asymptotic Integrability, in symmetry and Perturbation Theory," Symmetry and perturbation theory, vol. 1, pp. 2337, 1999.

[13] Tu, X. a. Yin and Zhaoyang, "Global weak solutions for a generalized Casmassa-Holm equation,"
Mathematische Nachrichten, vol. 291, 2018.

[14] Blaszak and Maciej, The Theory of Hamiltonian and Bi-Hamiltonian Systems. In: Multi-Hamiltonian Theory of Dynamical Systems., Berlin: Springer, 1998, pp. 41-85. 